\title{
Massive separation control analysis of the pulsed jet actuators effects
}

\author{
P. Gillieron ${ }^{1, a}$ And A. Kourta ${ }^{2}$ \\ 1 Chercheur indépendant \\ 2 Laboratoire PRISME, Université d'Orléans, France
}

Received 18 June 2013, Accepted 13 December 2013

\begin{abstract}
The aerodynamic drag of simplified car geometry with small span and strongly separated wake can be reduced up $20 \%$ by using pulsed jet distributed along the rear window. Results show an enhancement of transversal wake size in the control region, a displacement of the mean positions of vortex wake structures, a decrease of velocities near the rear part and a reduction of vortex structure recirculation in the wake. These modifications produced by the control are analyzed, commented and model based on simple strategy to build efficient and suitable separation control strategy is proposed.
\end{abstract}

Key words: Aerodynamic / control / actuator / pulsed jet / modeling

Résumé - Contrôle des décollements massifs, analyse des effets d'un actionneur de jets pulsés. La traînée aérodynamique d'une géométrie simplifiée de faible allongement à sillage fortement décollé peut être réduite de $20 \%$ à partir d'un contrôle par jets pulsés distribués sur le haut de la lunette arrière. Les résultats font apparaître un accroissement de la dimension transversale du sillage au niveau du contrôle, un report vers l'aval des positions moyennes des structures tourbillonnaires de sillage, une réduction des vitesses au voisinage de la partie arrière et une réduction de la circulation des structures tourbillonnaires transportées dans le sillage. Ces évolutions sont analysées, discutées puis modélisées à partir de considérations simples afin de mettre au point des protocoles de contrôle des décollements mieux adaptés et plus efficients.

Mots clés : Aérodynamique / contrôle / actionneur / jets pulsés / modélisation

\section{Introduction}

The aerodynamic drag coefficients of the road vehicles do not change since 30 years ago. These coefficients have been reduced by third between 1970th and 1985th under the pressure and with the help of the states as a consequence of oil crisis. Today the average of these coefficient values is approximately about 0.30 for the best [1]. Knowledge exists to reduce again by third these coefficients. However their applications still far from what the costumers wish to have. Hence the goal consists on to improve the actual vehicle shape in order to become more aerodynamic without modifying the external design.

One solution can be a distribution of orifices on the vehicle skin. These orifices will be used to blow or to suck air continuously or alternatively [2-4]. These solutions do not modify the vehicle geometry and could allow the design to be free from an important part of aerodynamic constraint that slow down the shape vehicle creativity.

\footnotetext{
a Corresponding author:

rdmfa.pgillieron@orange.fr
}

$20 \%$ drag reduction has been already obtained on simplified automotive vehicle geometry with a rear window tilted at $35^{\circ}[5,6]$. This reduction is obtained by using a set of pulsed jets placed at the top of the rear window. The control effects on the rear window separation and on the base are presented in this paper. In the base of the analysis and discussion by using simple considerations, model to obtain suitable and efficient control strategy is proposed.

\section{Experimental model}

The experiments has been conducted on the flow around a simplified model named Ahmed body [7] (Fig. 1). The roof is an horizontal flat surface and the rear window is a flat plate inclined in the bottom direction with an angle of $35^{\circ}$ from the horizontal plane. The link between roof and the top of the rear window is a continuum surface with a radius angle of $20 \times 10^{-3} \mathrm{~m}$. The same rounded surface is between the base and the lower surface of Ahmed body. These surfaces are not presented on Figure 1. 


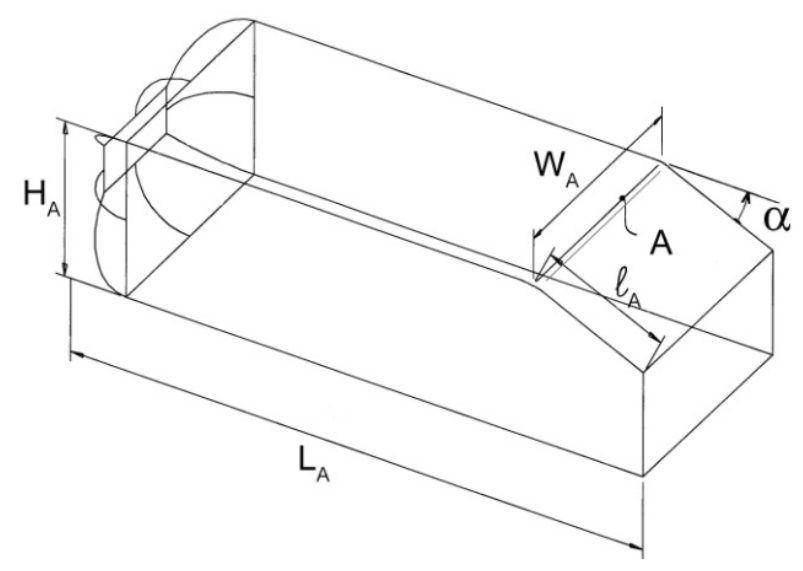

Fig. 1. Ahmed body model at size 0.7. The line A is the fictive junction between the roof and the top of the rear window [7].

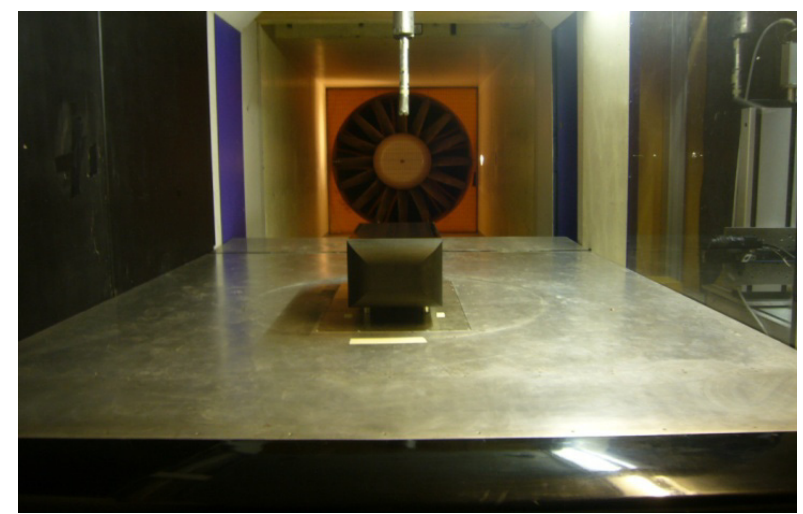

Fig. 2. Experimental model in the work section of the wind tunnel. The flow is oriented toward the fan located downstream of the model [5].

The model is equipped with a set of 5 actuators located at $5 \times 10^{-3} \mathrm{~m}$ from the top of the rear window. The slot size is equal to $0.5 \times 10^{-3} \mathrm{~m}$. Each actuator is able to pulse air at $0.5 \times 10^{-3} \mathrm{~m}^{3} . \mathrm{s}^{-1}$ with a maximum frequency of $550 \mathrm{~Hz}[5,6]$. Actuators are aligned transversally and their actuations are in phase and powered with compressed air. The pressure difference between the two sides of the actuators is in the range from 1.5 to $6 \times 10^{5} \mathrm{~Pa}$. The pulsed jet characteristics are determined by measuring the velocity field with hot wire in the longitudinal plane of symmetry of the model $(y=0)$.

\section{Experimental setup}

Experiments have been conducted on subsonic wind tunnel of PRISME laboratory of University of Orléans. The test section is $2 \mathrm{~m}$ high, $2 \mathrm{~m}$ wide and $5 \mathrm{~m}$ long. The maximum free stream velocity in the test-section is $60 \mathrm{~m} / \mathrm{s}$, the free stream turbulence intensity is below $0.4 \%$ and the mean flow homogeneity is $0.5 \%$ along a transverse distance of $1200 \mathrm{~mm}$. The model is installed on a 6-axis aerodynamic balance using 4 'feet' which are $20 \mathrm{~mm}$ in diameter cylindrical posts and are fixed to a horizontal

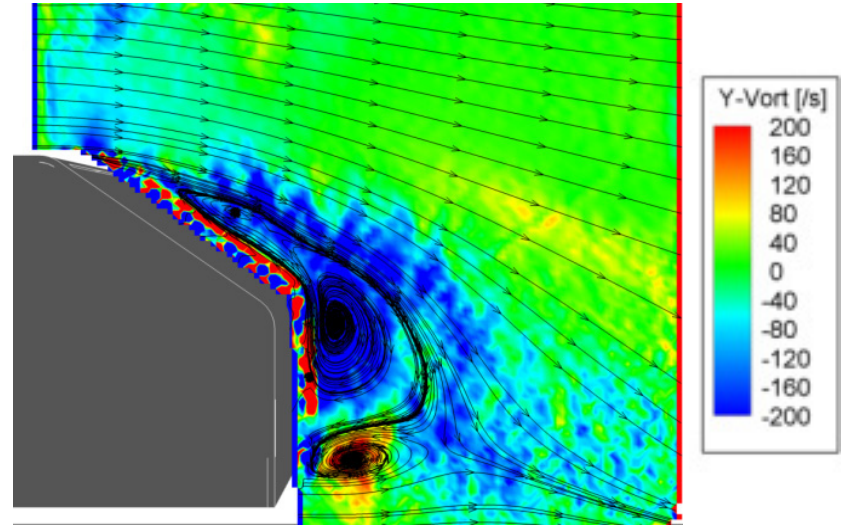

Fig. 3. Streamlines and vorticity field without control $[5,6]$.

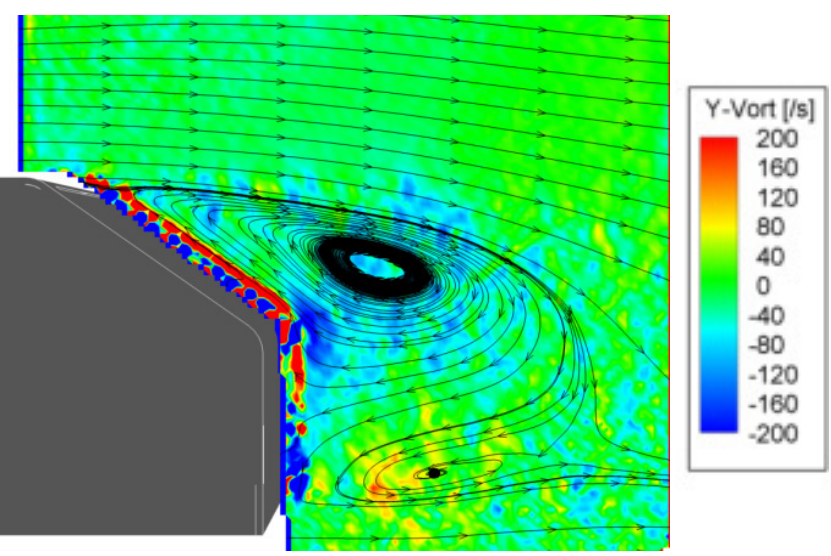

Fig. 4. Streamlines and vorticity field with control $[5,6]$.

metal frame that is connected to the balance. The model is placed above a $2 \mathrm{~m}$ wide and $3 \mathrm{~m}$ long flat plate located at $480 \mathrm{~mm}$ above the floor of the wind tunnel. The leading edge of the flat plate is elliptic and its trailing edge is controllable in order to suppress the longitudinal pressure gradient in the test section. The ground clearance is $0.07 \mathrm{~m}$ between the Ahmed body and the surface of a flat plate. In this work the inlet velocity is equal to $30 \mathrm{~m} . \mathrm{s}^{-1}$ and the Reynolds number based on the length of the model is $1.4 \times 10^{6}$.

\section{Results}

The highest drag reduction obtained of $20 \%$ [6] comes with an enhancement of the transversal size of the wake at the top of the rear window (Figs. 3 and 4), a downstream displacement of mean vortex structure positions in the wake (Figs. 3 and 4), a reduction of the wake top and bottom structure vorticity $\omega_{y}$ (Tab. 1) and a global decrease of the velocity near the base and the rear window as can be observed in the parietal visualizations (Figs. 5, $6)$. These results are the effects of the pulsed jet generated by the actuators and suggest initializing discussion to better understand the control effects. 
Table 1. $x_{m} / H_{A}, z_{m} / H_{A}$ are non dimensional coordinates and $\omega_{y}$ the vorticity is in the $y$ direction. $H_{A}$ is the height of the body used in the experiment.

\begin{tabular}{|l|ccc|ccc|}
\cline { 2 - 7 } \multicolumn{1}{c|}{} & \multicolumn{2}{c|}{ Vortex structure from rear window } & \multicolumn{2}{c|}{ Vortex structure from underbody } \\
\cline { 2 - 7 } \multicolumn{1}{c|}{} & $x_{m} / H_{A}$ & $z_{m} / H_{A}$ & $\omega_{\mathrm{y}}\left({\left.\mathrm{rd} . \mathrm{s}^{-1}\right)}^{-1} x_{m} / H_{A}\right.$ & $z_{m} / H_{A}$ & $\omega_{y}\left(\mathrm{rd}^{-1}\right)$ \\
\hline Without Control & 0.14 & 0.50 & -290 & 0.20 & 0.05 & 193 \\
With Control & 0.30 & 0.75 & -92 & 0.49 & 0.10 & 54 \\
\hline
\end{tabular}

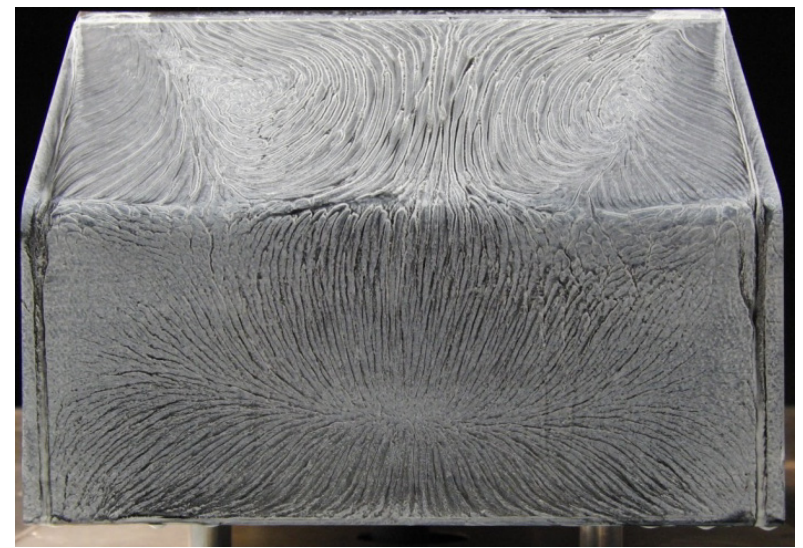

Fig. 5. Case without control, wall visualization in the base and in the rear window [6].

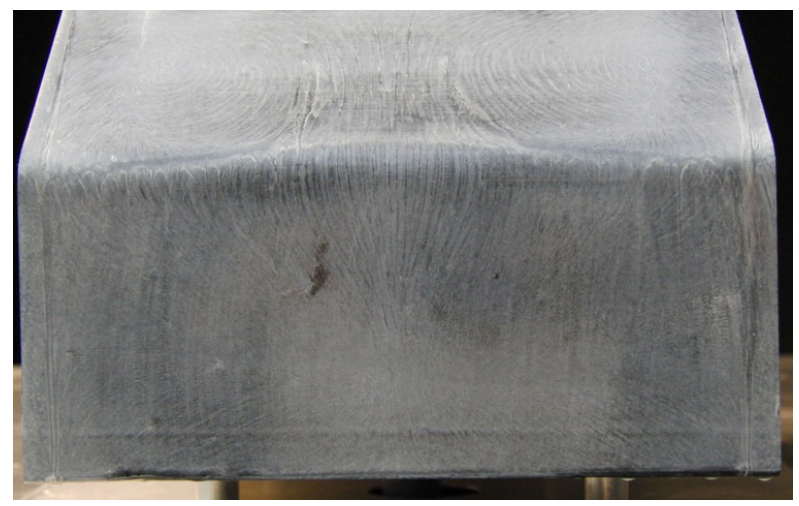

Fig. 6. Case with control, wall visualization in the base and in the rear window $[6]$.

\section{Control effect analysis}

In the following discussion, jet considered is plane and continuous along the slot span and the end effects are neglected. At any position and outside of potential region, the velocity modulus is dependent on the time and the distance $\mathrm{d}$ from the jet section reference (located upstream and at the jet axis located in the median plane to the outlet plane). At each time $t$, the evolution with the position is given by an equation as the inverse of the mean square of the distance $d[8]$. Its evolution is given by:

$$
\frac{V_{m}(d)}{U_{o}}=2.68 \sqrt{\frac{e}{d}}
$$

In this equation, $V_{m}(d)$ is the maximum velocity in the median plane perpendicular to the actuator outlet plane,

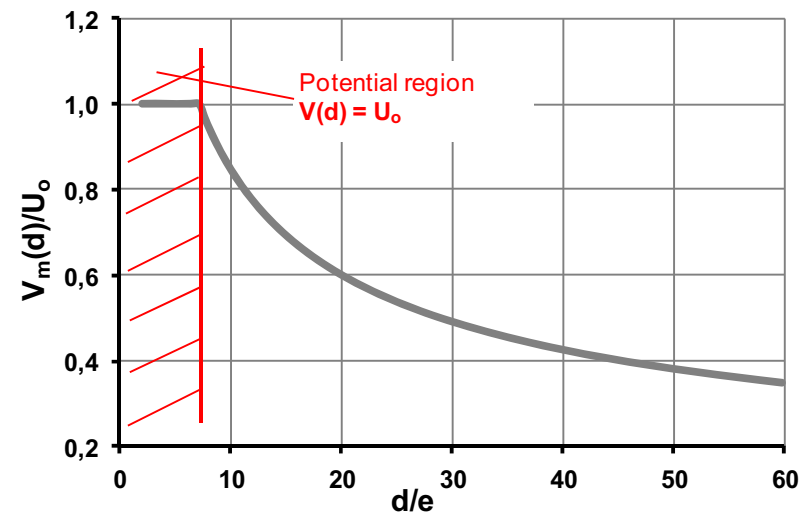

Fig. 7. Evolution of the reduced maximum velocity $V_{m}(d) / U_{\text {o }}$ in the middle plane normal to the actuator exit versus the reduced distance $d / e$, following Abramovich (plane jet theory) [8].

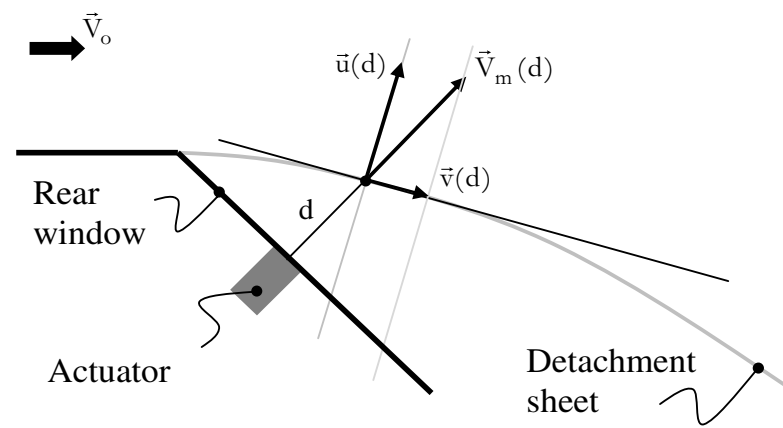

Fig. 8. Plane jet actuator placed at the top of the rear window [6]. The jet is normal to the rear window surface. The jet velocity is named $V_{m}(d), d$ is the distance from the rear.

$U_{\mathrm{o}}$ is the momentum velocity at the actuator outlet and $e$, the height (in the longitudinal direction). The variation of the reduced velocity $\frac{V_{m}(d)}{U_{o}}$ with a non-dimensional distance $\frac{d}{e}$ is plotted in Figure 7 .

Near the separated layer, the jet velocity has normal and tangential component named $v(d)$ and $u(d)$, as presented in Figure 8. The effects of these components on the separated shear layer circulation are analyzed in the following parts.

\subsection{Increase of the wake vertical size}

When the separated shear layer moves towards the slot, the normal jet velocity component $u(d)$ reduces the vertical motion of the shear layer and the displacement 
amplitude toward the rear window decreases (see Fig. 8). The opposite mechanism appears when the shear layer moves far from the slot. And hence the jet tends to reduce the displacement of the shear layer toward the rear window. As far as the jet does not cross the separated shear layer the continuous or pulsed blowing tends to move the vortex centers towards the wake edges [1] and consequently reduce the pressure drag at the base [9]. The flow is subsonic, the increase of the pressure at the base affects the pressure upstream and modifies the pressure distribution along the body surface. These modifications allow to obtain $20 \%$ drag reduction. The same analysis can be in the flow coming from the bottom side.

\subsection{Downstream displacement of the vortex structures positions}

The tangential jet component $v(d)$ increases locally the transport velocity of the vortex structures coming from the rear window. The structure is transported rapidly downstream, its mean position calculated over the same period moves far from the base and induced velocities near the base decrease. This evolution can be clearly observed on wall visualization of Figures 5 and 6 . This induced velocity reduction provides an increase of the base pressure and hence the pressure drag at the base decreases $[1,9]$. As indicated previously this pressure drag evolution at the base modifies the wall distribution of the pressure and of the shear stress on the body surface and leads to $20 \%$ aerodynamic drag reduction.

The vortex structures issued from the roof and the bottom of the body interact together. This interaction, dependent on the distance between these two structures, decreases when this distance increases. The induced velocity of the vortex structure issued from the top on the one coming from the bottom oriented upstream decreases however the velocity transport of the vortex coming from the top increases. The displacement velocity of the bottom vortex structure increases and hence this structure moves downstream more quickly (see Tab. 1).

To these effects on the vertical size of the wake and on the displacement velocity of the vortex structures, the circulation (and the rotational) of vortices contributes also significantly.

\subsection{Effect of the circulation of the wake structures}

If $\Delta v$ refers to a velocity variation over the jet axis projected on the tangent to a separated layer between $d-\varepsilon$ et $d+\varepsilon$, the components of the velocity coming from the pulsed jet projected on the local tangent to the separated sheet on both sides of the sheet are given by, see Figures 9 and 10:

$$
\begin{aligned}
& v^{+}=v(d)-\Delta v \\
& v^{-}=v(d)+\Delta v \quad \text { with } \quad \Delta v>0
\end{aligned}
$$

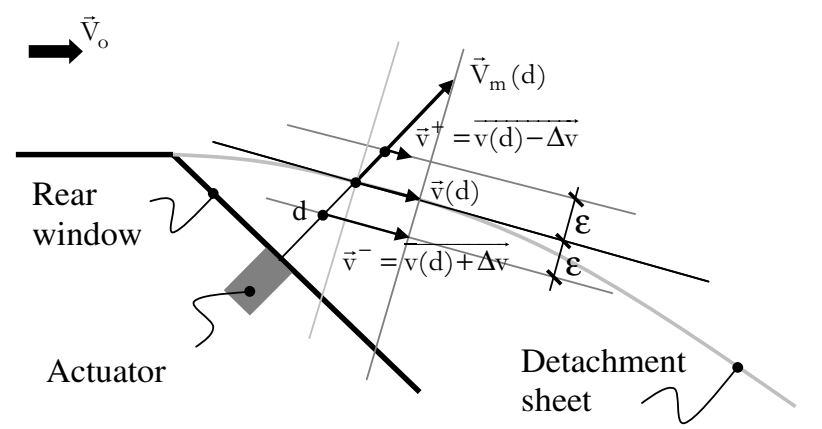

Fig. 9. Separated sheet and components of the velocity coming from the pulsed jet projected on both sides of the sheet.

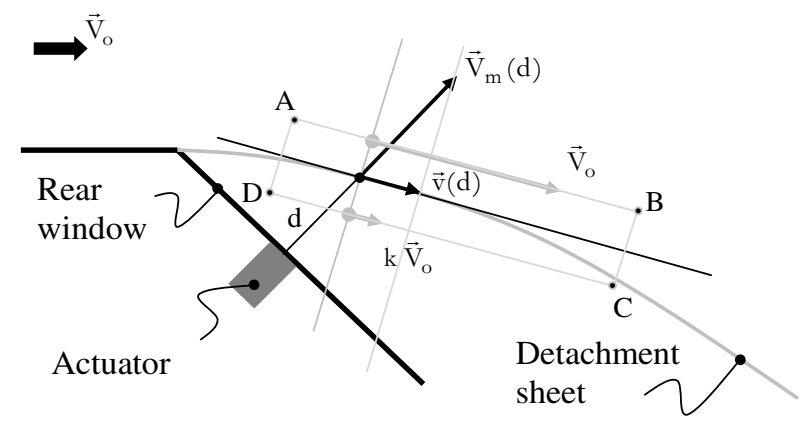

Fig. 10. Velocities on both sides of the sheet around the closed contour ABCD.

On both sides of the separated sheet, the transport velocities are:

$$
\begin{aligned}
& V_{R}^{+}=V_{o}+v^{+} \\
& V_{R}^{-}=k V_{o}+v^{-} \quad \text { with } \quad k<1 \quad \text { close to } 0.1 \text { à } 0.2
\end{aligned}
$$

These two relations associated to equations (2) and (3) lead to:

$$
\begin{aligned}
& V_{R}^{+}=V_{o}+v(d)-\Delta v \\
& V_{R}^{-}=k V_{o}+v(d)+\Delta v
\end{aligned}
$$

Along a closed contour (ABCD) with a length of $\Delta l$ and a thickness of $2 \varepsilon$, the elementary circulation $\left(\Delta^{2} \Gamma_{c}\right)$, in the case where the control is on, is given by, see Figure 10:

$$
\begin{aligned}
\Delta^{2} \Gamma_{c}= & \left(V_{o}+v(d)-\Delta v\right) \Delta l-\left(k V_{o}+v(d)+\Delta v\right) \Delta l \\
= & \left(V_{o} \Delta l-k V_{o} \Delta l\right)-2 \Delta v \Delta l \\
& \text { with } \Delta l>0 \text { and } \varepsilon \rightarrow 0 \\
= & \Delta^{2} \Gamma_{w} \quad \text { (without control) }-2 \Delta v \Delta l
\end{aligned}
$$

And the circulation of velocity vector at both sides of the separated shear layer of the rear window in the control case is smaller than the one without control. Following Stokes theorem, to this recirculation reduction is associated a velocity rotational decrease and hence the one of vortex structures in the wake. This effect linked to previous effects (Sects. 5.1 and 5.2) can has an important effect on the wall pressure and shear stress distribution on the body and modifies the rotational and frequency of the vortex shedding. These effects can partly explain the experimental obtained drag reduction. 


\section{Conclusion}

Results associated to $20 \%$ drag reduction obtained by pulsed jet control applied to a rounded rear window tilted at $35^{\circ}$ of a simplified geometry have been analyzed, discussed and a model based on simple consideration is proposed. These considerations allow to explain and to characterize each evolution observed in the wind tunnel experiments.

The increase of the separation vertical size observed on the top of the rear window and the downstream displacement of the vortex structure mean positions are associated the normal and tangential velocities induced by the jet. The increase of the vortex structure velocity transport coming from the top of the rear window in the flow direction modifies the flow field velocity induced by the vortex structures coming from the body bottom, explains the downstream displacement of the vortex structures mean positions and the increase of the base wall pressure. A circulation model allows to explain the vortex structure rotational reduction obtained experimentally.

By acting on the kinematic of the wake, the control modifies the wall pressure and shear stress distributions on the complete body geometry and generates evolutions in energy dissipation per unit process. When these evolutions allow reducing energy dissipation per unit, the drag reduces and this reduction can be important. This analysis and improvement of understanding of the control effects on the evolution of the pressure and shear stress wall distribution, in the origin of aerodynamic drag, have to be continued.

\section{References}

[1] P. Gilliéron, A. Kourta, Aérodynamique automobile pour l'environnement, le design et la sécurité, Editions CEPADUES, ISBN No. 978.2.85428.969.5, 2011

[2] S. Aubrun, J. MacNally, F. Alvi, A. Kourta, Separation flow control on a generic ground vehicle using steady microjet arrays, Experiments in Fluids, DOI10.1007/ s00348-011-1132-0

[3] P. Joseph, X. Amandolèse, J.L. Aider, Drag reduction on the $25^{\circ}$ slant angle Ahmed reference body using pulsed jet, Experiments in Fluids 52 (2012) 1169-1185

[4] M. Pastoor, L. Henning, B.R. Noack, R. King, G. Tadmor, Feedback shear layer control for bluff body drag reduction, J. Fluid Mech. 608 (2008) 161-196

[5] E. Bideaux, P. Bobillier, E. Fournier, P. Gilliéron, M. El Hajem, J.Y. Champagne, P. Gilotte, A. Kourta, Aerodynamics for land vehicles; Flow control; Drag reduction with pulsed jets on thick body and massive flow separation, IJAD, 2010 Indersciences Enterprises Ltd, 2011, pp. 282-298

[6] P. Gilliéron, A. Kourta, Aerodynamic drag control by pulsed jets on simplified car geometry, Exp. in Fluids 54 (2013) 14-57

[7] S.R. Ahmed, R. Ramm, G. Flatin, Some salient features of the time averaged ground vehicle wake, SAE technical paper Series 840300, 1984

[8] G.N. Abramovich, Teoriia turbulentnykh strui. Moscow, 1960

[9] C.H. Bruneau, E. Creusé, D. Depeyras, P. Gilliéron, I. Mortazavi, An analytical approach to improve drag control techniques for the Ahmed body, Joint Fluids Engineering Conference, 2011 